Informes de la Construcción Vol. 25, nº 243

Agosto - septiembre de 1972

Con este artículo finaliza una corta serie que hemos ido publicando, en diferentes números de INFORMES, correspondientes a otras tantas ponencias presentadas en el I Congreso Nacional de la Calidad, celebrado en Madrid en febrero pasado. El autor expone que gran parte de los edificios que actualmente se construyen tienen estructura resistente de hormigón armado, lo cual exige un perfecto conocimiento de sus condiciones de resistencia y de la forma de fabricarlo adecuadamente. Se recuerdan los preceptos de la Instrucción (Presidencia del Gobierno de 20 de septiembre de 1968) y se hace hincapié en la importancia de que, cuantos intervienen en proyectos y construcciones de hormigón, conozcan las definiciones de la resistencia característica y del coeficiente de variación de las muestras, así como de la dispersión o variabilidad y la necesidad de su control mediante gráficos, cuyos trabajo y costo son precisos y menores de lo que tal vez pueda creerse, dejando que la experiencia haga lo que falta. 


\section{el control de la calidad en el hormigón}

PEDRO MENDIZABAL LARUMBE, Dr. Ingeniero Industrial Asesor Estadístico de los Laboratorios de Ensayos e Investigación Industrial «L. J. Torróntegui», de Bilbao

De los materiales que hoy se emplean en la construcción, uno de los más importantes es el hormigón, armado y sin armar.

El hormigón armado constituye la estructura resistente de la mayor parte de los edificios que en la actualidad se erigen, por lo que es necesario especificar sus condiciones de resistencia y fabricarlo de modo que cumpla con esas condiciones establecidas.

En teoría, la estructura de hormigón armado está diseñada para que el hormigón trabaje a compresión y el hierro a tracción; y aunque en ocasiones se le exija también alguna otra característica, es indudable que la condición más importante del hormigón es su resistencia a la compresión.

Pero, ¿cómo medir la resistencia a la compresión del hormigón de una estructura construida?

La técnica ha establecido que se saquen muestras de las hormigoneras al hacer la obra, que se hagan probetas de forma y dimensiones ya definidas, se mantengan esas probetas durante 28 días en condiciones determinadas y se rompan sometiéndolas a compresión en prensas de ensayo preparadas para ese fin.

En estos ensayos se obtienen unos números que difieren entre sí y de los que hay que deducir la resistencia que se toma como base para el cálculo. Esta decisión no es fácil y escapa al técnico individual.

Como nuestro conocimiento de las cosas es imperfecto, el conjunto de científicos, ingenieros y técnicos debe establecer unas normas con arreglo a los conocimientos existentes en el momento, a los que todos nos hemos de sujetar. La responsabilidad personal desaparece al realizar lo que el mundo manda en ese momento. 
En nuestro país existe una Instrucción aprobada por Decreto de la Presidencia del Gobierno, de 20 de septiembre de 1968, a la que han de ajustarse cuantas obras se realicen en el territorio nacional (1).

Para proyectar una estructura el técnico debe partir de la tensión máxima que puede admitirse en cualquier punto, lo que está en relación directa con lo que se ha llamado «resistencia característica».

La Instrucción dice, en su artículo $10 .^{\circ}$ Hormigones, página 27:

\section{«10.1. Resistencia del hormigón a compresión}

Se define como resistencia característica de un hormigón en obra el valor que se obtiene, a partir de una serie de $n$ ensayos de resistencia sobre probetas, al multiplicar por 2 la media aritmética de los $n / 2$ resultados más bajos y restar después la media aritmética del conjunto de los $n$ resultados. Si el número $n$ es impar se prescindirá para lo anterior del valor mediano de la serie.»

Es decir, que si hemos obtenido, al romper 6 probetas los valores 220, 213, 200, 197, 231,222 , la resistencia característica será:

$$
\begin{gathered}
\sigma_{b k}^{\prime}=\frac{197+200+213}{3} \times 2-\frac{220+213+200+197+231+222}{6}= \\
=\frac{610}{3} \times 2-\frac{1.283}{6}=406,7-213,8=192,9 .
\end{gathered}
$$

En los comentarios al artículo 10., dice la Instrucción: «La definición adoptada corresponde, aproximadamente, a un grado de confianza del $95 \%$, es decir, que existe una probabilidad del $5 \%$ de que el hormigón (supuesto representado por una serie de probetas cuyas cargas de rotura se distribuyen según una ley estadística normal) rompa con una tensión inferior a su resistencia característica. Dicha definición no es más que una simplificación práctica de la expresión matemática siguiente, de cálculo más complicado:

$$
\sigma_{b k}^{\prime}=\sigma_{b m}^{\prime}(1-1,64 \delta)
$$

donde $\sigma_{b m}^{\prime}$ es la resistencia media y $\delta$ es el coeficiente de dispersión (desviación cuadrática media relativa) arrojada por los ensayos.»

Es de observar que el Comité Europeo del Hormigón, en el que España tiene representantes colaborando con los de otras naciones, define «R.2.12, pág. 113:

(1) Todavía no es obligatoria; pero no tiene duda que, en un litigio por defecto de construcción, el fallo podría considerar las recomendaciones de esa Instrucción. 


\section{Resistencia característica del hormigón}

El valor de la resistencia característica a la compresión $\sigma_{b k}^{\prime}$ (o a la tracción $\sigma_{b k}^{\prime}$ ) es el que corresponde a la probabilidad de tener en una distribución característica normal de los resultados de medidas a la compresión (o a la tracción) $5 \%$ de los resultados inferiores al valor así fijado.»

Y en los comentarios:

«CR 2.12:

Esta elección corresponde a adoptar el valor 1,64 para el coeficiente $\chi$. La resistencia característica está así determinada por la fórmula:

$$
\sigma_{b k}^{\prime}=\sigma_{b m}^{\prime}-1,64 s=\sigma_{b m}^{\prime}(1-1,64 \delta) \text {. }
$$

Una proximación que conduce sensiblemente al mismo resultado pero que evita el cálculo de las desviaciones cuadráticas, consiste en tomar para valor de la resistencia característica dos veces la media de la mitad de los resultados inferiores a la mediana, disminuido de la media de los resultados.»

Queda bien claro que la definición de la resistencia característica está definida por la fórmula:

$$
\sigma_{b k}^{\prime}=\sigma_{b m}^{\prime}(1-1,64 \delta)
$$

y que la definición que se da en la Instrucción Española no es más que la forma de tener un resultado aproximado al que daría la fórmula, evitando el cálculo de $\delta$.

Antes de seguir, se ha de advertir que a $\delta$ el Comité Europeo del Hormigón y la Instrucción Española le llaman desviación cuadrática media relativa, cuando ese parámetro estadístico se llama tanto en francés como en español «Coeficiente de Variación» (1) y se designa con la letra $V$; así le llamaremos en lo sucesivo.

Es verdaderamente importante que cuantos intervienen en proyectos y en construcciones de hormigón conozcan y comprendan la definición de la resistencia característica y se sensibilicen a la importancia del coeficiente de variación.

Con respecto a la importancia de la variabilidad en el hormigón se decía ya en la Instrucción h.a.61 del Instituto Torroja del año 1961, bajo el título «A.j.17 Resistencia característica»:

«Sea $n$ el número de probetas ensayadas y rotas a compresión... A primera vista el criterio más lógico para definir la resistencia de semejante material es el de identificar su resistencia característica con la resistencia media $R_{m}$ :

$$
R_{m}=\frac{1}{n} \sum_{i=1}^{i=n} R_{i}
$$

(1) En el Glosario publicado por la Organización Europea del Hormigón, en el que aparecen los términos estadísticos en doce idiomas, figura con ese nombre. 
de las $n$ probetas ensayadas. Este criterio presenta la ventaja de que en la formación de $R_{m}$ intervienen todas las cargas de rotura registradas, difuminándose con las restantes el posible error que se pudiera introducir en la estimación de uno de los valores observados.

Por el contrario, ofrece el inconveniente de no reflejar la dispersión que registran los resultados individuales. Un hormigón que, por determinadas circunstancias, presenta unas resistencias sumamente variables y discordantes, puede incluso ofrecer un elevado valor medio de su carga de rotura sin que dicha media aritmética $R_{m}$ acierte a expresar la poca confianza que cabe depositar en un material de tan desigual comportamiento.»

Estas atinadas consideraciones nos han inducido a utilizar la importante información de que se dispone en los Laboratorios de Olaveaga, por haberse roto miles de probetas desde su fundación, para exponer con más claridad la importancia de la dispersión o variabilidad y la necesidad de su control.

Para precisar la influencia de esa dispersión vamos a hacer unos números.

Sea $R_{m}$ la resistencia media de las probetas rotas.

$R_{c}$ la resistencia característica especificada $\left(120 \mathrm{kp} / \mathrm{cm}^{2}\right.$, como mínimo, según la Instrucción vigente).

$s$ la desviación standar de los resultados obtenidos.

$V$ el coeficiente de variación, que está definido por la relación:

$$
V=\frac{s}{R_{m}} .
$$

La fórmula que define la resistencia característica es:

$$
R_{c}=R_{m}(1-1,64 \mathrm{~V}) \text {; }
$$

con $R_{c}=120$, se tiene:

T A B L A I

$\begin{array}{lcccccccccc}V & = & 10 & 12 & 14 & 16 & 18 & 20 & 22 & 25 & 30 \\ R_{m}= & 144 & 149 & 156 & 163 & 170 & 179 & 188 & 203 & 222\end{array}$

Las antiguas normas h.a.61 consideraban como valor medio del coeficiente de variación 0,166 , e incluso este valor lo habían incluido en una fórmula. Con este coeficiente de variación, para tener $R_{c}=120$ es preciso $R_{m}=165$.

Nosotros, con información de numerosas obras de importancia muy diversa, hemos de observar que en nuestro país el coeficiente de variación medio se encuentra entre 0,20 y 0,25 , dándose con frecuencia valores de 0,30 y aún mayores.

Además se presenta a veces una gran diferencia en el coeficiente de variación en el transcurso de la obra o en las diferentes partes de la misma.

De una obra bastante importante realizada hace pocos años, tomamos del resumen que se hizo: «Se ha calculado el coeficiente de variación del conjunto, pero tiene muy poco valor: En columnas, $29,4 \%$; en vigas, $24,7 \% . »$ 
En ese análisis se decía que tenía poco valor ese coeficiente de variación conjunto, porque variaba muchísimo de una parte a otra de la obra.

De unos cuantos valores comprendidos entre 15 y 20 se pasaba a valores de 30 y hasta $38,4 \%$. Naturalmente, la resistencia media parecía admisible; la resistencia característica calculada por la fórmula $R_{c}=R_{m}(1-1,64 \mathrm{~V})$ era inferior en muchos puntos a $90 \mathrm{kp} / \mathrm{cm}^{2}$, llegando en un lugar a su valor mínimo de $60,4 \mathrm{kp} / \mathrm{cm}^{2}$, cifra alarmante.

Para el cálculo de la estructura, la Instrucción Española establece un coeficiente de mayoración para las cargas que depende de varias circunstancias a considerar y cuyo valor medio es 1,50. Establece también un «coeficiente de minoración de la resistencia del hormigón» que lo toma igual a 1,50, es decir, que bajo las solicitaciones de trabajo se puede suponer, claro está que solamente desde un punto de vista de aproximación, que el coeficiente de trabajo del hormigón será $R_{c} / 1,5 \times 1,5$ y que el coeficiente de seguridad global relativo del hormigón es de $1,5 \times 1,5=2,25$. Tomando para la resistencia característica el valor de $180 \mathrm{kp} / \mathrm{cm}^{2}$, por ejemplo, se obtendría una tensión aproximada de trabajo de $0,8 \times 180 / 2,25=64 \mathrm{kp} / \mathrm{cm}^{2}$, habiendo tenido en cuenta el coeficiente de cansancio. En el caso antes citado podía ser la tensión de trabajo superior a la resistencia.

En América, según las normas del American Concrete Institute, se admite como esfuerzo máximo admisible al que puede estar sujeta una pieza $0,45 R_{c}$, siendo $R_{c}$ el valor especificado de la resistencia definida en forma semejante a la que aquí se cita. La única diferencia es que clasifica el hormigón en dos grupos. En la primera clase admite que una probeta cada diez se rompa por debajo del valor especificado, y en la segunda, dos cada diez.

Como la definición americana de la que podemos llamar resistencia característica admite que una probeta de cada diez se rompa por debajo de ese valor, parece ligeramente más benévola la norma americana que la europea con muy poca diferencia. Sin embargo, para juzgar sobre ello habría que tener en cuenta todos los aspectos del cálculo.

Podíamos poner muchos ejemplos revisando nuestro archivo de los 13 años transcurridos en estos Laboratorios en el que hay registrado algún coeficiente de variación por encima de 0,45 , pero no es necesario insistir para comprender que es preciso, si se quiere que se nos considere como un país en progreso, llevar a cabo un control de la resistencia media $R_{m}$ y del coeficiente de variación $V$.

Vamos, ahora, a ver la posibilidad y forma de hacerlo.

\section{Gráficos de control}

Es el método más práctico y eficaz de efectuar el control de la media y del coeficiente de variación de las muestras; pero el control del coeficiente de variación se puede realizar indirectamente mediante el gráfico de control del recorrido de las muestras.

En una muestra de $n$ unidades, el recorrido está definido por la diferencia entre el valor mayor y el menor de los hallados al inspeccionar las $n$ unidades de la muestra.

En la distribución normal se verifica que:

$$
s(\text { desviación standard })=\frac{\bar{R}}{d_{2}} ;
$$


siendo $R$ el promedio de los recorridos hallados en muestras sucesivas y $d_{2}$ un coeficiente cuyos valores son:

TA B L A I I

$\begin{array}{lccccccccc}n= & 2 & 3 & 4 & 5 & 6 & 7 & 8 & 9 & 10 \\ d_{2}= & 1.128 & 1.693 & 2.059 & 2.326 & 2.534 & 2.704 & 2.847 & 2.970 & 3.078\end{array}$

$y$ puesto que

$$
V=\frac{s}{R_{m}}=\frac{\bar{R}}{d_{2} \cdot R_{m}}
$$

una vez que se ha fijado el número de unidades de la muestra, si se controla $R$, indirectamente queda controlado $V$.

En la misma hoja de papel se pueden llevar tres gráficos.

En el primero (fig. 1) se consigna en el eje horizontal el número de orden de la muestra y en la vertical correspondiente los seis valores que se han hallado y su promedio. Se traza una línea horizontal en el valor de $R_{c}$ y se puede trazar una línea en el de $R_{m}$ que corresponda a un valor de $V$, que el constructor crea debe mantener. Con una tilde se han señalado los valores correspondientes a las seis probetas de la muestra y con un círculo el valor de la media.

Si el valor de $R_{m}$ es exactamente el teórico y se conserva $V$, el 5, \%, aproximadamente, de los valores individuales se encontrarán por debajo de $R_{c}$.

Para observar bien la media $R_{m}$, es recomendable llevar otro gráfico, llamado gráfico móvil de medias (fig. 2). Como antes, en el eje horizontal se consigna el número de la muestra y en la línea vertical correspondiente se anota la media de los promedios de cinco muestras consecutivas, es decir, se empieza en el número 5 , donde se anota:

$$
\frac{R_{m_{1}}+R_{m_{2}}+R_{m_{3}}+R_{m_{4}}+R_{m 5}}{5}
$$

A la vertical número 6 se lleva el valor:

$$
\frac{R_{m_{2}}+R_{m_{3}}+R_{m_{4}}+R_{m 5}+R_{m_{6}}}{5}
$$

y así sucesivamente. Para cada vertical se quita el primer valor de la anterior y se le añade el que corresponde al número de la muestra. Este gráfico es más sensible que el primero para comprobar las tendencias del promedio a subir o bajar.

Por último se lleva el gráfico móvil del recorrido $R$ (fig. 3), que indica la tendencia del recorrido. Se hace en la misma forma que el anterior. En el número 5 se ha anotado:

$$
\frac{R_{1}+R_{2}+R_{3}+R_{4}+R_{5}}{5} .
$$


figuras

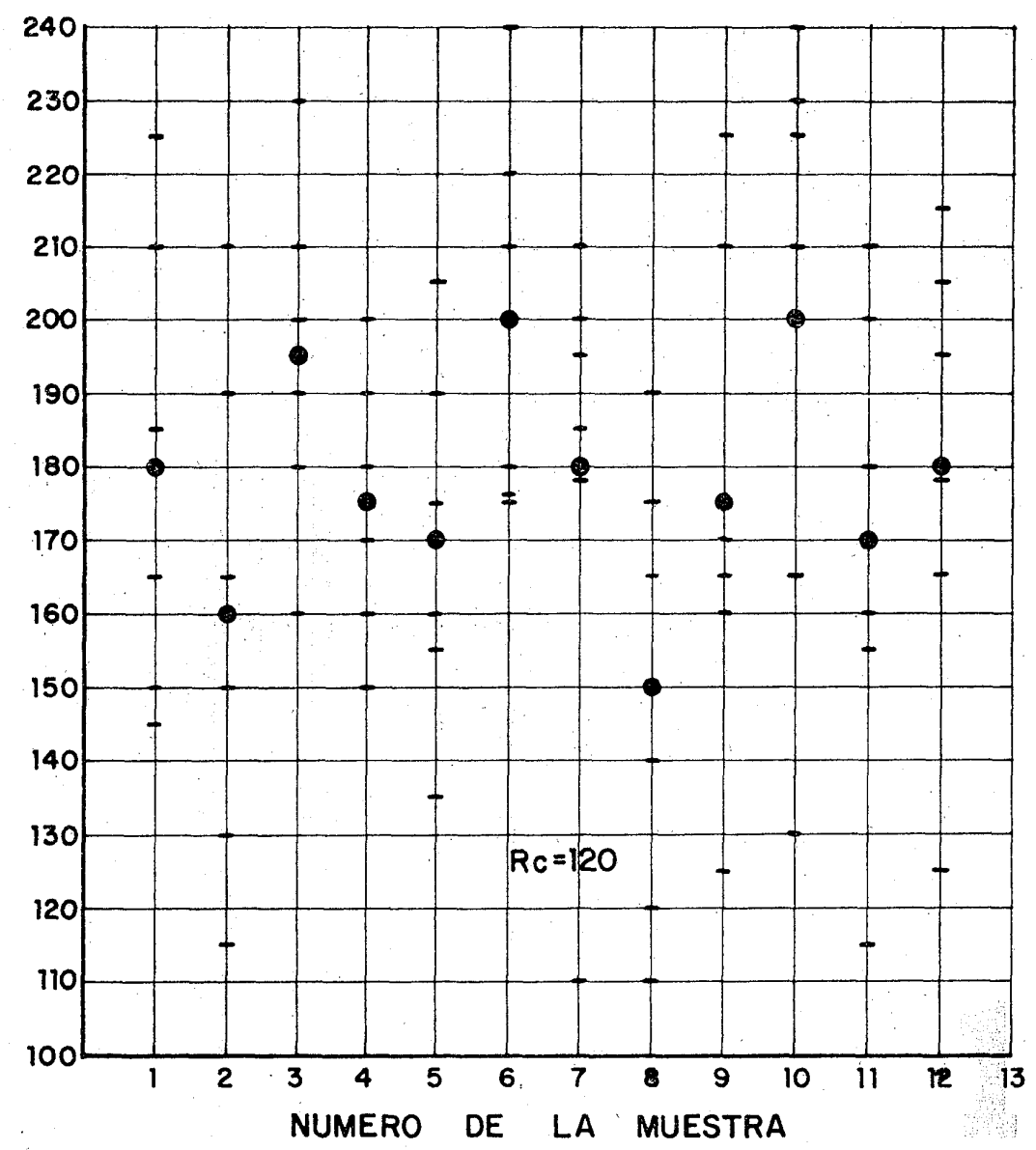

Gráfico de promedios - Valores individuales $-(n=6)$
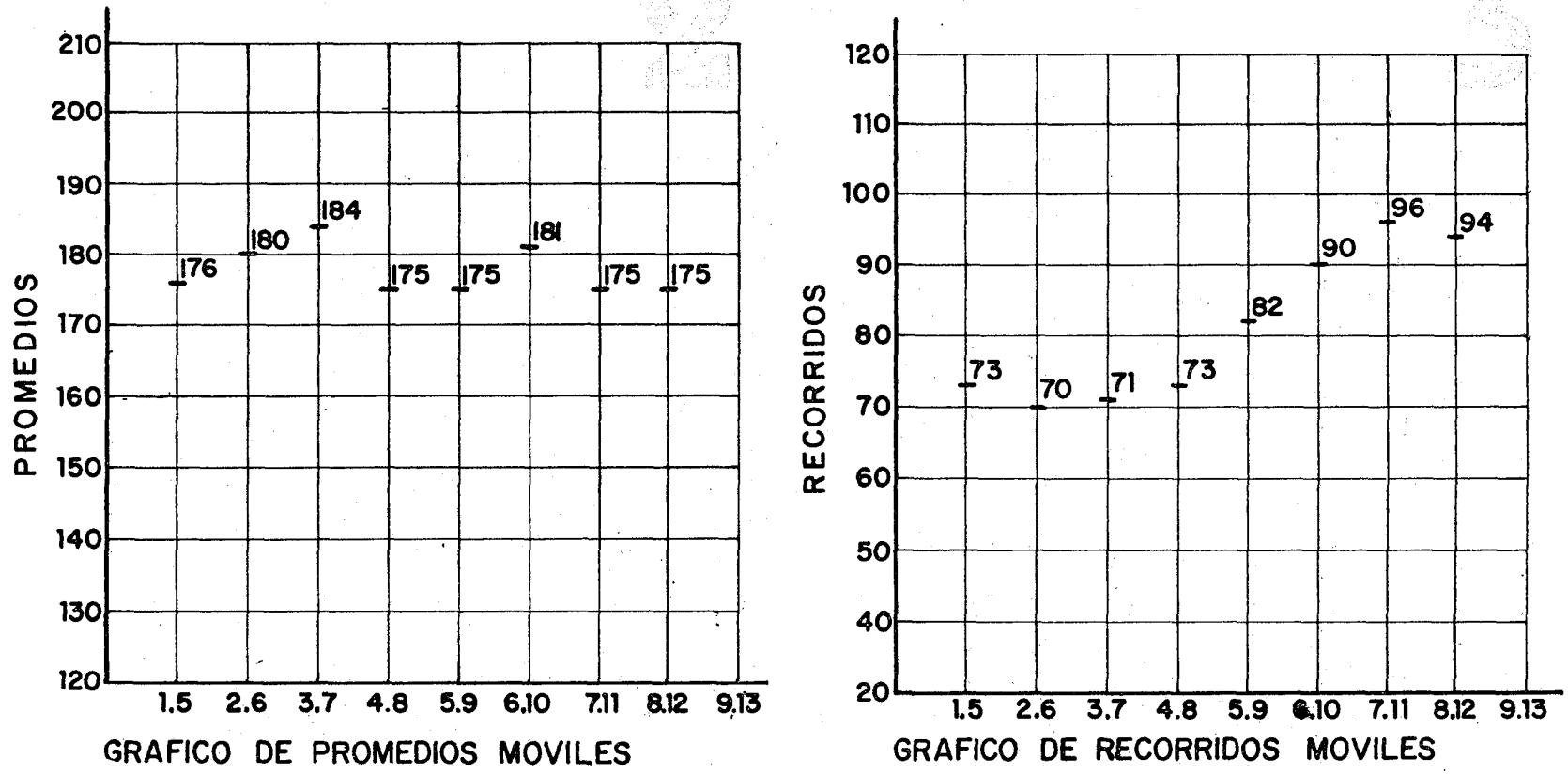
En el 6:

$$
\frac{R_{2}+R_{3}+R_{4}+R_{5}+R_{6}}{5}
$$

y así sucesivamente.

Ahora con los promedios móviles de $R_{m}$ y $R$ se determina el coeficiente de variación por la expresión

$$
V=\frac{\bar{R}}{d_{2} \cdot R_{m}}
$$

en la que $\bar{R}$ es el promedio de cinco recorridos y $d_{2}$ viene dado en la tabla II, viéndose que al aumentar $R$, aumenta $V$ y que, por tanto, si en el gráfico móvil del recorrido se ve que éste aumenta, es forzoso aumentar también el promedio, como se ve en la tabla I. Ahora bien, si $n$, el número de unidades de la muestra se conserva constante, se puede poner esa tabla en función del recorrido.

De la relación $V=\frac{\bar{R}}{d_{2} \cdot R_{m}}, \quad \bar{R}=d_{2} \cdot V \cdot R_{m}$.

Sustituyendo los valores en al tabla I obtenemos:

\begin{tabular}{|c|c|c|c|c|c|c|c|c|c|}
\hline$R_{m}=$ & 144 & 149 & 156 & 163 & 170 & 179 & 188 & 203 & 222 \\
\hline$n=6 \bar{R}=$ & 36,5 & 45,3 & 55,3 & 66,1 & 77,5 & 90,7 & 104,8 & 128,6 & 168,8 \\
\hline
\end{tabular}

TA B L A I I I

El primer valor es $\bar{R}=2,534 \times 0,10 \times 144=36,5$.

La tabla III tiene un pequeño inconveniente. No sirve más que para muestras de 6 unidades, porque el coeficiente $d_{2}$ cambia con el número de unidades de la muestra, pero se puede preparar fácilmente para cualquier valor de $n$.

Como se verá, da muy poco trabajo llevar estos gráficos.

El verdadero trabajo está en la toma de muestras y en la preparación, fraguado y rotura de las probetas. Llevar los números a los gráficos no representa trabajo apreciable.

En cambio, requiere un especial cuidado la toma de muestras.

No se debe olvidar que la información que se obtiene de unas muestras no vale más que las muestras que la han proporcionado. Si para hacer las probetas se pone más cuidado en la amasada o se falsea el procedimiento de control no se ha conseguido nada. Aún peor; se puede creer que se está haciendo bien cuando en realidad van mal las cosas. Es necesario que la muestra sea representativa del hormigón que se está produciendo. Como es lógico, habrá más dispersión entre amasadas que dentro de una amasada y habrá aún mayor en el transcurso del tiempo.

Es preciso que la toma de muestras se haga en forma totalmente aleatoria. Recomendamos la lectura de "Sampling Fresh Concrete», norma C 172 de la ASTM, pág. 128, de la parte 10 de la edición de 1969. 
Con todo lo antedicho no se pretende resolver el problema del control de la calidad del hormigón, problema complejo y en el que deben intervenir cuantos a la construcción se dedican. Se ha pretendido llamar la atención sobre el asunto y apuntar una posible solución, pero es el conjunto corporativo el que debe dar instrucciones sobre la forma de realizarlo. La experiencia hará lo demás.

Ha de tenerse en cuenta la cuestión económica. Tomar la muestra, preparar las probetas, conservarlas y romperlas tiene un coste que hay que afrontar. Sin embargo, en la mayor parte de los casos más que problema económico es de atención y de intención.

Como el control cuesta dinero, los constructores en general quieren hacer ver solamente lo que les cuesta, pero hay que pensar que es rentable, porque evita gastos de reparación y daños que se pueden ocasionar. El equilibrio perfecto económico es difícil alcanzar, aunque se puede asegurar que es caro no llevar ningún control.

Por último, aunque sea un poco ajeno a la cuestión que aquí se examina, nos parece que ha pasado ya bastante tiempo desde 1968 para que el período de prueba de la Instrucción de aquel año pueda darse como satisfactoriamente terminado.

He de agradecer la eficaz colaboración que para este trabajo me han prestado D. José Luis Ramírez y D. José Mendía, del Departamento de Construcción de los Laboratorios de Ensayos e Investigación Industrial «L. José de Torróntegui», anexos a la Escuela Técnica Superior de Ingenieros Industriales de Bilbao.

\section{résumé}

Le contrôle de la qualité du béton

Pedro Mendizábal Larumbe, Dr. ingénieur industriel

Cet article met fin à une courte série d'articles insérés en différents numéros de cette revue, correspondant à autan d'autres rapports présentés lors du le. à Madrid au mois de février dernier.

L'auteur expose qu'une grande partie des édifices en cours de construction ont une structure porteuse en béton armé, ce qui exige une connaissance parfaite de ses conditions de résistance et de la manière de l'exécuter convenablement. II rappelle les normes éta blies par la Présidence du Gouverne ment espagnol, le 20 septembre 1968 et met en évidence l'importance qu'ont pour tous ceux qui interviennent dans la conception et exécution d'ouvrage en béton, la connaissance des définitions de la résistance caractéristique et du coefficient de variation des échan tillons, ainsi que de la dispersion ou variabilité, et la nécessité de leur con trôle par des graphiques, dont le travail et coût sont nécessaires et moins im portants que ion ne le pourrait penser peut-être, laissant à l'expérience la so lution des autres problèmes.

\section{summary}

Quality control in concrete

Pedro Mendizábal Larumbe, Dr. of Industrial Engineering

This article is the last of a series which we have been publishing in various issues of INFORMES, dealing with the papers presented at the First National Congress on Quality, held in Madrid last February.

The author explains that most of the buildings being constructed at present have a resistant frame of reinforced of its resistance requirements is essential as well as its suitable manufacture. He mentions the Instruction Regulations of 20 September 1968, and emphasizes how important it is that all those in volved in designing and building with lding with concrete be familiar with the definitions of its characteristic resistance, and the variation coefficient of the samples, as well as the dispersion and variability, and the necessity of its control by means of graphs. Experience shows that this makes for less work and lower cost than is probably Imagined.

\section{zusammenfassung}

Die Güteüberwachung des Betons

Pedro Mendizábal Larumbe, Dr. ing.

Mit diesem Aufsatz wird die Kurze Serie beendet, die in verschiedenen Nummern dieser Zeitschrift erschienen st, in der einige der Bertchte, die im 1. Ien nationalen acute-Kongress" gehal geben wurden.

Der Verfasser bemerkt, dass die meisten heutigen Hochbauten eine Stah betonstruktur haben, was genaue Kennt nisse seiner Festigkeitsbedingungen und der angemessenen Herstellungsverfaht erfordet. Es wird auf die Vorschrifte der Richtlinien (Eriass des Regierungsvorsitzender des 20 September 1968) hingewiesen und es wird ebenfalls besonders betont, dass alle die am Entwurf und der Bauausfunrung beteiligt sind die Begriffe der Festigkeit und Variationsspanne der Proben kennen, ebenso wie die Streuung und die Erforderlichkeit sie mittels Graphiken zu Uberwachen, wobei die Arbeit und Kosten nötig sind und viel niedriger als man oft annimmt. 